\title{
Strain and Grain Connectivity in Bi2223/Ag Superconducting Tapes
}

\author{
Ying Kai Huang, Bennie ten Haken and Herman H. J. ten Kate \\ University of Twente, Faculty of Applied Physics, P. O. Box 217,7500 AE Enschede, The Netherlands
}

\begin{abstract}
The critical current reduction in silver-sheathed $(\mathrm{Bi}, \mathrm{Pb})_{2} \mathrm{Sr}_{2} \mathrm{Ca}_{2} \mathrm{Cu}_{3} \mathrm{O}_{10}$ superconducting tapes (Bi2223/Ag) is investigated when loaded with uni-axial strains in combination with a magnetic field perpendicular to the tape surface. The number and quality of the grain-to-grain connections and the alignment of the superconducting cores mainly determine the critical current in $\mathrm{Bi} 2223 / \mathrm{Ag}$ tapes. It is assumed that the transport current flows simultaneously through two current carrying paths in the tape: one through the network of Josephson junctions and the other is through the well-connected grains. The model describes well the magnetic field dependence of the critical current at various strains. A detailed analysis has shown that strain deteriorates grain connectivity, induces cracking and hence changes the current carrying path. Furthermore, strain may introduce new defects inside the grains along the strong-link current path and increase intra-granular pinning strength.
\end{abstract}

\section{INTRODUCTION}

Superconducting materials for industrial scale applications must fulfill the requirements in superconducting properties as well as mechanical properties. One of the important design parameters is critical current density as a function of magnetic field and strain: $J_{\mathrm{C}}(T, B, \varepsilon)$. If strain changes the critical current of a superconductor, two mechanisms can be considered. One is by changing the intrinsic properties of the material, i.e. the pinning force and the critical parameters $B_{c 2}$ and $T_{c}$. Another is by changing the geometry such as the conducting area and current path, which affects the overall current carrying capability. These considerations hold true also for the high $T_{C}$ superconducting composite such as Bi2223/Ag tape.

The strain dependence of critical current of $\mathrm{Bi} 2223 / \mathrm{Ag}$ tapes has been investigated extensively [1-5]. It is generally agreed that strain causes an irreversible reduction of the critical current of a tape. The critical current of a Bi2223/Ag tape is closely related to the grain connectivity and the alignment of grains. It is assumed that two different currentcarrying paths exist in the tape. One is the Josephson junction network formed by weakly linked grains; the other is through the well-connected grains, such as those connected by a lowangle $a b$-axis grain boundary or the well aligned thin layers in the vicinity of the silver-filament interfaces. These two current carrying paths can be distinguished due to their different magnetic field dependences [6]. It is interesting to investigate how these two current carrying paths are affected by strain. In this paper, the results are presented and discussed for the reduction of the critical current of Bi2223/Ag tapes under a uni-axial compressive or tensile strain in combination with a magnetic field perpendicular to the tape surface.

Manuscript received September 15, 1998

\section{EXPERIMENTAL}

Non-twisted $\mathrm{Bi} 2223 / \mathrm{Ag}$ tapes with 85 filaments, fabricated by "oxide powder in tube" method [7], are used in the experiments. The dc $V-I$ characteristics of the tapes at $77 \mathrm{~K}$ as a function of axial strain and magnetic field are measured using the four-point configuration in the "bending-spring" strain set-up [2]. Uni-axial strain, ranging from $-1 \%$ to $+1 \%$, can be realized in this set-up. The tape is soldered to the brass substrate of the bending spring. Two pieces of the same tape are required for the investigation in compressive and tensile strain regime, respectively. DC magnetic fields up to $0.4 \mathrm{~T}$. are applied perpendicular to the broad face of the tape. The critical currents are determined using a voltage criterion of $10^{-4} \mathrm{~V} / \mathrm{m}$.

\section{RESULTS AND DISCUSSION}

The critical current $I_{\mathrm{C}}$ of the $\mathrm{Bi} 2223 / \mathrm{Ag}$ tapes at $77 \mathrm{~K}$ as a function of axial strain and dc magnetic field is shown in Figs. 1 and 2. The main features of the strain and magnetic field effects are summarized as follows.

When a tensile strain is applied, $I_{\mathrm{C}}$ first decreases slightly with the strain up to about $0.4 \%$. Further increase of the tensile strain results in a dramatic reduction of $I_{\mathrm{C}}$. In a $0.3 \%$ strain increment, $I_{\mathrm{C}}$ drops to a value less than $60 \%$ of its original value.

The magnetic field does not seem to change the general characters of the strain effect on the critical current of the tapes. However, if the critical currents at different strains are normalized to the "zero-field" critical current $I_{C}(0, \varepsilon)$ and plotted against the magnetic field (Fig. 3), some interesting features are revealed. When $\varepsilon \leq 0.4 \%$, the normalized $I_{\mathrm{C}}(B, \varepsilon)$ curves are about the same. As the tensile strain is further

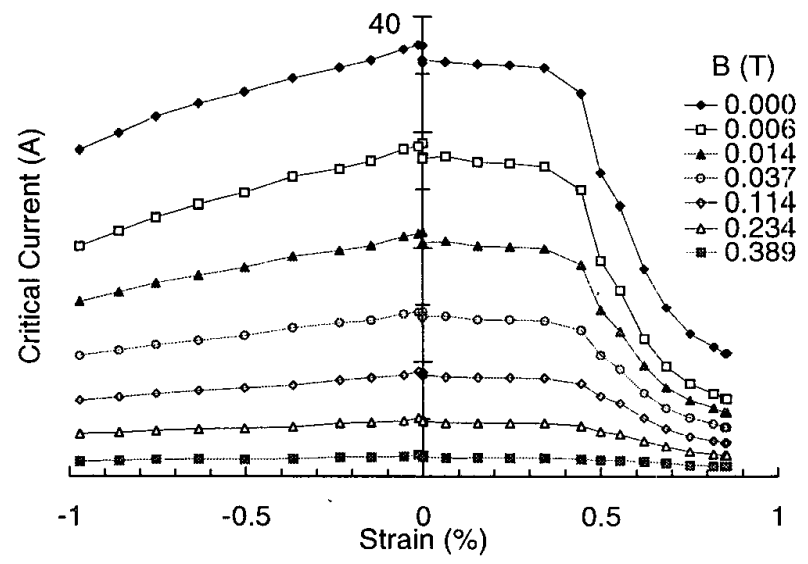

Fig. 1. Critical current of $\mathrm{Bi}-2223 / \mathrm{Ag}$ tapes at $77 \mathrm{~K}$ as a function of compressive and tensile strain at various dc magnetic fields perpendicular to the tane surface. 


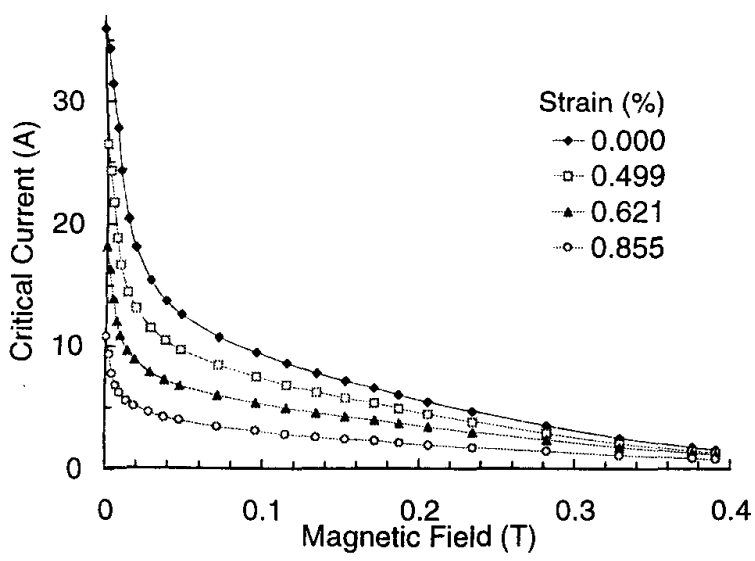

Fig. 2. Critical current of $\mathrm{Bi}-2223 / \mathrm{Ag}$ tapes at $77 \mathrm{~K}$ as a function of $\mathrm{dc}$ magnetic field at different tensile strains. The magnetic field is applied perpendicular to the tape surface.

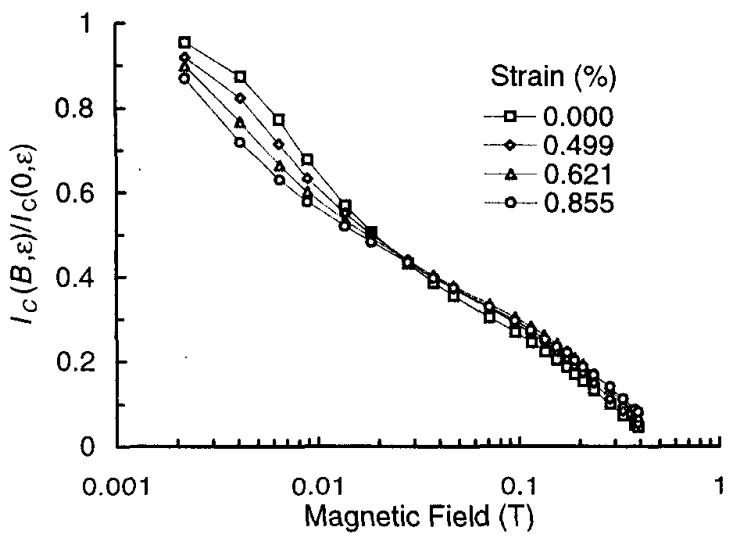

Fig. 3. Critical currents $I_{C}(B, \varepsilon)$ of the $B i 2223 / \mathrm{Ag}$ tape at $77 \mathrm{~K}$ at various magnetic fields and tensile strains normalized to the critical currents at zero magnetic field $I_{C}(0, \varepsilon)$, indicating that when strain is increased the relative reduction of $I_{\mathrm{C}}$ is faster at low fields and becomes opposite at higher fields.

increased, the $I_{\mathrm{C}}(B, \varepsilon)$ for $B<28 \mathrm{mT}$ reduces relatively quicker than that for $\varepsilon=0 \%$. This becomes more pronounced with the increase of the strain. At higher fields, $I_{\mathrm{C}}$ decreases relatively slower and imposes to a new curve for $\varepsilon>0.7 \%$.

When a compressive strain is applied, $I_{\mathrm{C}}$ decreases monotonically in the entire strain range measured. The reduction of $I_{\mathrm{C}}$ in the range of $-0.7 \%<\varepsilon \leq 0 \%$ can be described by a constant relative slope $C(B)$, i.e.

$$
I_{\mathrm{C}}(B, \varepsilon)=I_{\mathrm{C}}(B, 0) \exp [C(B) \varepsilon] .
$$

The magnetic field dependence of the relative slope $C(B)$ is shown in Fig. 4. With the increase of the magnetic field, $C(B)$ increases at very low field and passes an maximum at about $8 \mathrm{mT}$. When the field is higher than $90 \mathrm{mT}, C(B)$ increases nearly proportional to $B$.

The reduction of $I_{C}$ deviates from $E q$. (1) when the compressive strain is further increased $(-1 \%<\varepsilon<-0.7 \%)$. The $I_{\mathrm{C}}$ decreases faster than that predicted by Eq. (1) when $B<90 \mathrm{mT}$ and is slower when $B>90 \mathrm{mT}$.

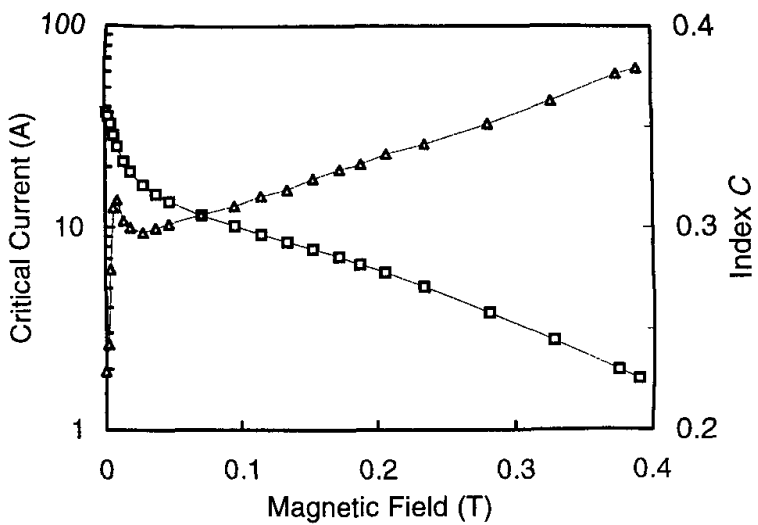

Fig. 4. Critical current $I_{C}$ (square) at zero strain and the magnetic field dependence of the relative slope $C$ (triangle) for the decrease of $I_{C}$ under compressive strains $(-0.7 \%<\varepsilon \leq 0 \%)$ at $77 \mathrm{~K}$.

The results presented above indicate that the strain has a certain effect on the $I_{\mathrm{C}}(B)$ characteristics of $\mathrm{Bi} 2223 / \mathrm{Ag}$ tapes, especially in the low field range. As Bi2223 is a fragile and granular material, the deformation of the tape may have two possible mechanisms. One is by changing the inter-granular links without breaking the superconducting junction between the grains. The other is by cracking the superconducting grains and grain boundaries and reducing the total cross section for transporting current. In order to understand the mechanism for the $I_{\mathrm{C}}$ reduction by strain, the following model is used to analyze the $I_{\mathrm{C}}(B, \varepsilon)$ curves.

The transport current is supposed to flow through two different paths in a tape simultaneously. One is through the weakly linked grain boundaries (Josephson junctions network), $I_{\mathrm{C} w}$, and the other through well-connected grains, $I_{\mathrm{Cs}}$, i.e.

$$
I_{\mathrm{C}}=I_{\mathrm{Cw}}+I_{\mathrm{Cs}}
$$

The $I_{\mathrm{Cw}}$ and $I_{\mathrm{Cs}}$ can be distinguished because of their different magnetic field dependence. For a Josephson junction, the Josephson current between adjacent grains in a local field has a characteristic of the Fraunhofer-pattern:

$$
I_{J}\left(B, B_{0}\right)=I_{0 w}\left|\frac{\operatorname{Sin}\left[\pi\left(B+B_{m}\right) / B_{0}\right]}{\pi\left(B+B_{m}\right) / B_{0}}\right| \text {, }
$$

where $B$ is the applied magnetic field and $B_{\mathrm{m}}$ is a field representing the conservation of magnetic history in the sample and the effect of self-field due to the transport current. $B_{0}$ is the characteristic field, $B_{0}=\Phi_{0} /(2 \lambda+d) w$, where $\Phi_{0}$ is the flux quantum, $\lambda$ the magnetic penetration depth, $d$ and $w$ the thickness and width of the junction.

The critical current along the weak-links path $\left(I_{\mathrm{CW}}\right)$ is the sum of the Josephson currents along the network of the Josephson junctions in a tape. Each of the Josephson junctions has a certain characteristic field $B_{0}$ depending on the dimension of the junction. The distribution of the characteristic field $B_{0}$ is related to the dimension distribution of the grain boundaries. The distribution function, $G\left(B_{0}\right)$, is assumed to be a log-normal one in the analysis: 


$$
G\left(B_{0}\right)=\frac{1}{\sqrt{2 \pi} \ln \sigma} \exp \left[-\frac{\left(\ln B_{0}-\ln B_{w}\right)^{2}}{2 \ln ^{2} \sigma}\right],
$$

where $B_{\mathrm{w}}$ and $\sigma$ are parameters representing the most probable $B_{0}$ and the width of the distribution, respectively. With this distribution function the $I_{\mathrm{Cw}}$ can be expressed as:

$$
I_{C w}(B)=\int_{a}^{b} I_{J}\left(B, B_{0}\right) G\left(B_{0}\right) d B_{0} / \int_{a}^{b} G\left(B_{0}\right) d B_{0} .
$$

The critical current carried by the strongly linked grains $\left(I_{\mathrm{Cs}}\right)$ is controlled by the flux pinning mechanism in the grains. The collective pinning theory [8] predicts an exponential dependence of $J_{C}(B)$. It is assumed that this can be expressed by a general form as proposed in [9]:

$$
I_{C s}(B)=I_{0 s} \exp \left[-\left(B / B_{s}\right)^{\alpha}\right],
$$

where $B_{\mathrm{s}}$ and $\alpha$ are related to the pinning characteristics of a tape.

All the $I_{C}(B, \varepsilon)$ curves can be described very well using the model above in the entire range of field measured $(B<0.4 \mathrm{~T})$, including the very low-field region where the self-field and remanent field are comparable to the applied field. One of the examples is given in Fig. 5.

A set of parameters used in Eqs. (2)-(6) for describing the experimental $I_{C}(B, \varepsilon)$ curves is obtained in the analysis. This is plotted as a function of strain in Fig. 6, excepting $B_{\mathrm{m}}$ and $\alpha$. The $B_{\mathrm{m}}$ in Eq. (3) varies from nearly zero to about $-0.7 \mathrm{mT}$. The index $\alpha$ in Eq. (6) is 1.4 for this tape and is kept constant for all strains.

It is shown that, before a strain is applied, the critical current attributed to the well-connected grains, $I_{\mathrm{Cs}}$, accounts for about $32 \%$ of the total critical current at zero applied field and becomes dominant in higher fields. The critical current attributed to the weakly linked grains, $I_{\mathrm{Cw}}$, decreases strongly in low-field range and becomes inversely proportional to the field at higher fields.

With the increase of a tensile strain, $I_{0 \mathrm{w}}$ remains constant for $\varepsilon<0.4 \%$ and decreases rapidly for higher strains. While $I_{0 \mathrm{w}}$ decreases monotonically with the increase of compressive

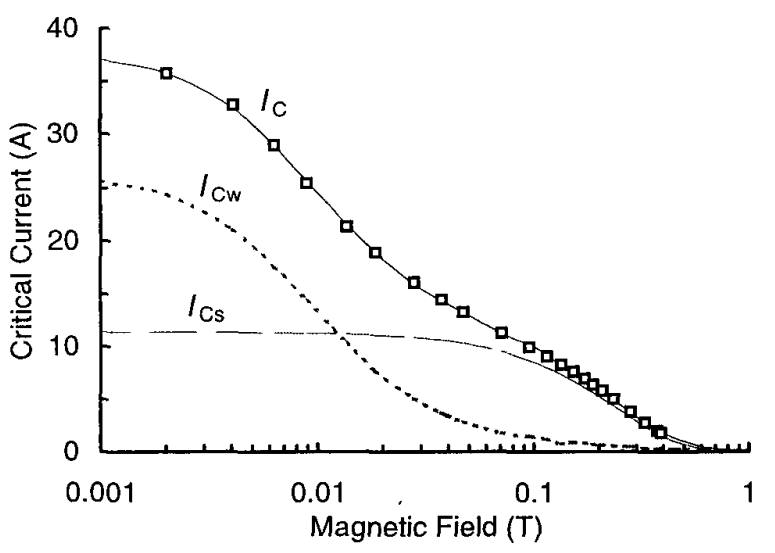

Fig. 5. Critical current of the Bi2223/Ag tape as a function of applied magnetic field. Open marks: measured $I_{C}$ at zero strain; Lines: calculation results according to Eas. (2)-(6).

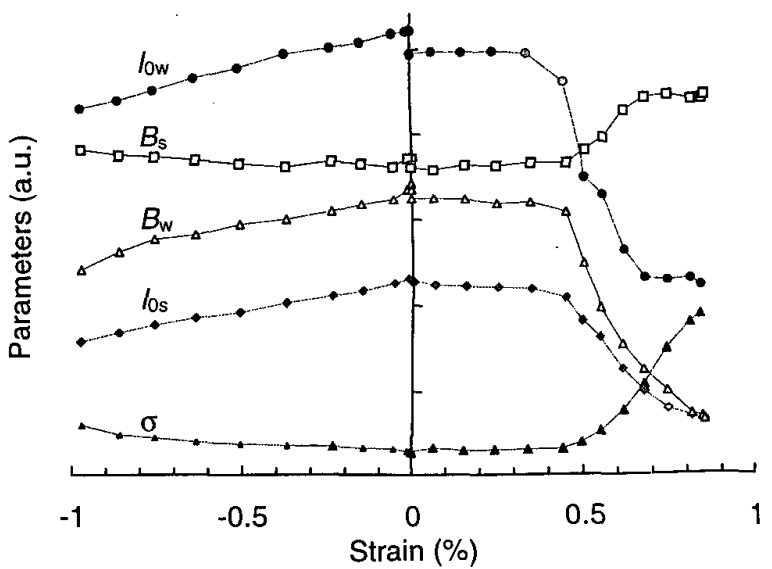

Fig. 6. Strain dependences of the parameters $I_{0 \mathrm{w}}, I_{(\mathrm{s}}, B_{s}, B_{w}$, and $\sigma$ for describing the experimental results of $I_{C}(B, \varepsilon)$ using Eqs. (2) - (6). The vertical axis is in an arbitrary unit, with a common zero at the lower end.

strain. The distribution of the characteristic field $B_{0}$ is broadened when strain is applied (Fig. 7). $B_{\mathrm{w}}$ shifts to lower value with strain ( $B_{\mathrm{w}}$ is about $17 \mathrm{mT}$ at $\varepsilon=0 \%$, and $12 \mathrm{mT}$ at $\varepsilon=-1 \%$ or $4 \mathrm{mT}$ at $\varepsilon=0.9 \%$ ). Because $B_{0}$ is inversely proportional to the thickness of a Josephson junction (a weakly linked grain boundary), the shift of $B_{\mathrm{w}}$ to lower value indicates that the grain connectivity in the tape is deteriorated. Under the influence of strain the currentcarrying capacity in this path is weakened and more sensitive to the presence of a magnetic field.

The normalized $I_{\mathrm{Cs}}(B, \varepsilon)$ and $I_{\mathrm{Cw}}(B, \varepsilon)$ to $I_{\mathrm{Cs}}(0, \varepsilon)$ and $I_{\mathrm{Cw}}(0, \varepsilon)$, respectively, as a function of magnetic field is shown in Fig. 8. How the strain affects both the strong-link and weak-link current-carrying path is clearly seen. As the strain increases, the reduction of $I_{\mathrm{Cw}}$ is relatively faster in the low field region due to the deterioration of grain connectivity. The reduction of $I_{\mathrm{Cs}}$ is relatively slower in the high field region (see the discussion later). These effects explain the experimental result shown in Fig.3.

Both $I_{0 \mathrm{w}}$ and $I_{0 \mathrm{~s}}$ are reduced with the increase of strain. This is due to the reduction of the effective cross section for transport current. Under tensile strain, the existing micro-

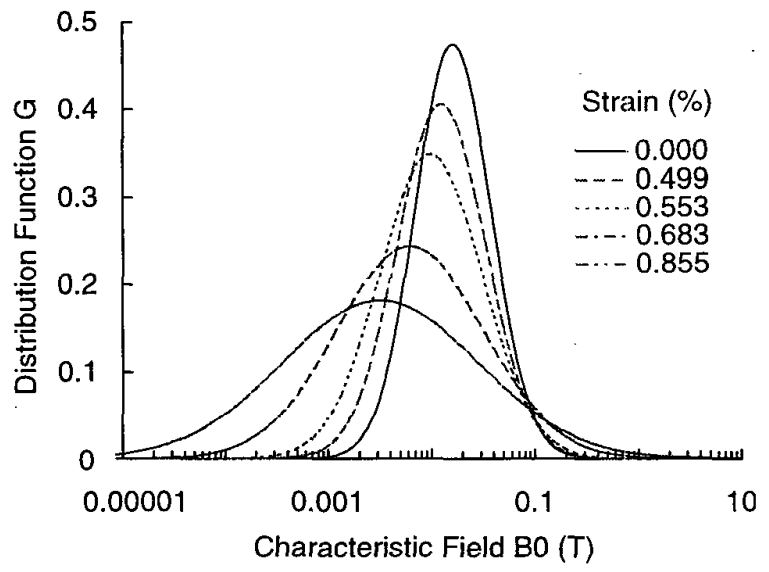

Fig. 7. Strain dependence of the characteristic field $B_{0}$ distribution (Eq. (4)) ir the $\mathrm{Bi} 2223 / \mathrm{Ag}$ tape. With the increase of strain, the most probable characteristic field $B_{\mathrm{w}}$ is shifted to a lower value and the distribution is broadened. 


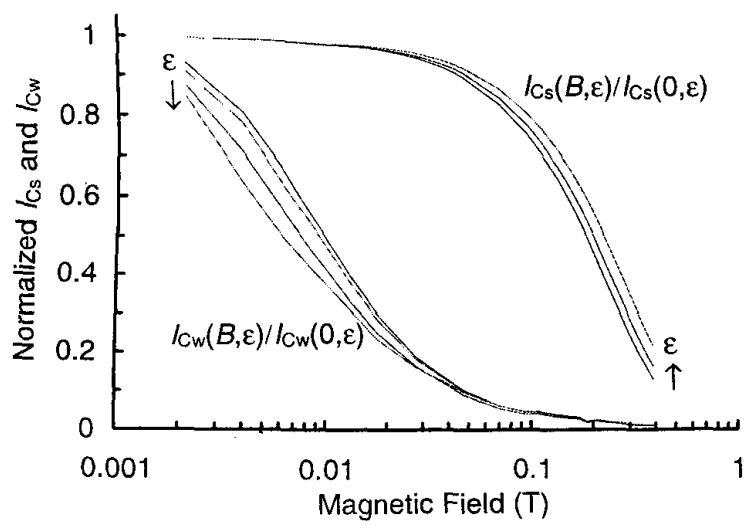

Fig. 8. Normalized $I_{\mathrm{Cs}}(B, \varepsilon)$ and $I_{\mathrm{Cw}}(B, \varepsilon)$ to $I_{\mathrm{Cs}}(0, \varepsilon)$ and $I_{\mathrm{Cw}}(0, \varepsilon)$, respectively, as a function of magnetic field, showing the strain effects on both the stronglink and weak-link current-carrying path, as compared with the experimental result shown in Fig.3.

cracks will spread rapidly to the entire thickness of the filament and new cracks are generated at the weakest locations in the filaments. This accounts for the dramatically reduction of the critical current at certain strains. The crack generation and propagation inevitably affect both the weakly linked and well-linked grain boundaries in the filaments, and hence alter the current carrying paths in the tape. The damage under a compressive strain is probably caused by sliding at grain boundaries [5]. The inter-granular bonding is fractured in shear since Bi2223 crystals have a (001) glide plane with very low stacking fault energy. This may be the reason why $I_{\mathrm{C}}$ is easily affected by compressive strain and the damage is also irreversible.

Another interesting effect of strain is the change of $B_{\mathrm{s}}$ in Eq. (6). $B_{\mathrm{s}}$ is about $0.24 \mathrm{~T}$ at $\varepsilon=0 \%$ and remains almost constant in the range of $-0.4 \%<\varepsilon<0.4 \%$. Further increasing the compressive strain, $B_{\mathrm{s}}$ increases slowly to a value of $0.25 \mathrm{~T}$ at $\varepsilon=-1 \%$. While further increasing the tensile strain, $B_{\mathrm{s}}$ increases to about $0.29 \mathrm{~T}$ at $\varepsilon \approx 0.7 \%$ and remains almost constant afterwards.

It is supposed that $I_{\mathrm{Cs}}$ in the strong-link current carrying path is controlled by collective flux pinning in grains and the pinning strength is reflected only by the $I_{\mathrm{Cs}}$ component in the tape [6], i.e.

$$
F_{\mathrm{p}}=I_{\mathrm{Cs}} B / S_{\mathrm{e}}=I_{0 \mathrm{~s}} B \exp \left[-\left(B / B_{\mathrm{s}}\right)^{\alpha}\right] / S_{\mathrm{e}},
$$

where $S_{\mathrm{e}}$ is the effective area for carrying the current $I_{\mathrm{Cs}}$. The uncertainty of $S_{\mathrm{e}}$ can be removed by normalizing $F_{\mathrm{p}}$ with the maximum pinning force. In this case, $B_{\mathrm{s}}$ is proportional to the peak field $B_{\mathrm{p}}$ at which the pinning force reaches a maximum.

According to the Kramer model for conventional type-II superconducting materials, the macroscopic pinning force is determined by two factors: one is the pinning strength for individual flux lines and the other is the shear strength of the flux line lattice. Flux motion occurs by flux-line depinning from pinning centers in the low-field region $\left(B<B_{\mathrm{p}}\right)$ and by synchronous shear of the flux-line lattice in the high-field region $\left(B>B_{\mathrm{p}}\right)$. A maximum of the pinning force appears where these two factors are approximately equal. $B_{\mathrm{s}}\left(\propto B_{\mathrm{p}}\right)$ is found to increase for certain strains (Fig. 6). This implies that the strain increases the shear strength of the flux line lattice in the grains. A possible explanation for this is that the strain introduces additional defects such as voids, stack faults or dislocations to the grains. The relatively slower decrease of $I_{\mathrm{C}}$ with the increase of strain at high magnetic fields as shown in Fig. 3 and Fig. 8 is probably related to the increase of $B_{\mathrm{s}}$ and therefore the pinning strength.

\section{CONCLUSION}

The reduction of the critical current of $\mathrm{Bi} 2223 / \mathrm{Ag}$ tapes under compressive or tensile strain is investigated in combination with dc magnetic fields applied perpendicular to the tape surface. The magnetic field dependence of $I_{\mathrm{C}}$ at various strains is well described by the model that distinguishes two co-existing current-carrying paths in the tape: one is through the Josephson junction network consisting of weakly linked grains; The other is through the strongly linked grains. Strain has certain effects on these two current carrying paths. It is concluded that, in addition to the strain introduced cracking process, strain deteriorates the grain connectivity, leading to the easier suppression of $I_{\mathrm{C}}$ by magnetic field in the low field region. For the well-connected current carrying path, strain on one hand, damages well connected grain boundaries through cracking or sliding. On the other hand, strain slightly increase the peak field of the maximum pinning force, indicating a certain increase of the shear strength of the flux line lattice in the grains.

\section{REFERENCES}

[1] J. W. Ekin, D. K. Finnemore and Qiang Li, J. Tenbrink and W. Carter, “ Effect of axial strain on the critical current of Ag-sheathed Bi-based superconductors in magnetic fields up to $25 \mathrm{~T}$," Appl. Phys. Lett. Vol. 61, pp. 858-860, August 1992.

[2] B. ten Haken, A. Godeke, H. J. Schuver and H. H. J. ten Kate, "Strain reduced critical current in $\mathrm{Bi}-2223 / \mathrm{Ag}$ superconductors under axial tension and compression," Adv. Cryo. Eng. 42, pp. 651-658, 1996.

[3] M. Suenaga, Y. Fukumoto, P. Halder, T. R. Thurston and U. Wildgrüber, "Effects of axial tensile and bending strains on critical currents of monoand multicored ( $\mathrm{Bi}, \mathrm{Pb})_{2} \mathrm{Sr}_{2} \mathrm{Ca}_{2} \mathrm{Cu}_{3} \mathrm{O}_{10}$-Ag tapes," Appl. Phys. Lett. Vol. 67, pp. 3025-3027, November 1995.

[4] M. R. Koblischka, T. H. Johansen and H. Bratsberg, "Bending of silversheathed $(\mathrm{Bi}, \mathrm{Pb})-2223$ tapes investigated by magneto-optical flux visualization," Supercond. Sci. Technol. Vol. 10, pp. 693-701, 1997.

[5] M. Lahtinen, J. Paasi, Z. Han and J. Sarkaniemi, "Microstructure of the ceramic in superconducting $\mathrm{Bi}-2223$ composite tapes after bending strain cycles," Physica C 277, pp. 238-242, 1997.

[6] Y. K. Huang, B. ten Haken and H. H. J. ten Kate, "Critical current of high Tc superconducting Bi2223/Ag tapes," submitted to Physica C.

[7] Q. Li, G. N. Riley, Jr., R. D. Parrella, S. Fleshler, M. W. Rupich, W. L. Carter, J. O. Willis, J. Y. Coulter, J. F. Bingert, V. K. Sikka, J. A. Parrell, and D. C. Larbalestier, "Progress in superconducting performance of rolled multifilamentary Bi-2223 HTS composite conductors," IEEE Trans. Appl. Superconductivity, vol. 7, pp. 2026$2029,1997$.

[8] M.V. Feigel'man and V.M. Vinokur, "Thermal fluctuation of vortex lines, pinning, and creep in high- $T_{\mathrm{c}}$ superconductors," Phys. Rev. B 41, pp. 8986-8990, 1990.

[9] J. Horvat, S.X. Dou, H.K. Liu and R. Bhasale, "Critical currents through strong links in $\mathrm{Ag} / \mathrm{Bi}-\mathrm{Sr}-\mathrm{Ca}-\mathrm{Cu}-\mathrm{O}$ superconducting tapes," Physica $\mathrm{C}$, vol. 271 , pp. $51-58,1996$. 